\section{PTU-083 CAUDATE LOBE RESECTION FOR COLORECTAL LIVER METASTASES: A MATCHED PAIR ANALYSIS}

doi:10.1136/gutjnl-2012-302514c.83

G Marangoni, ${ }^{*}$ A Hakeem, R K Prasad, G J Toogood, J P A Lodge. HPB and Transplant Unit, St James' Hospital, Leeds, UK

Introduction Tumours located in the caudate lobe can be challenging due to the anatomical relation of segment I with the inferior vena cava (IVC), the presence of multiple small caudate veins, variable bile ducts anatomy and difficult exposure during surgery. Some groups reported increased morbidity, smaller resection margins and inferior outcomes. Aim of the study was to compare the group of patients who underwent caudate lobe resection (CLR) with a group of patients without caudate lobe excision (NCLR).

Methods Over a 16-year period, from November 1994 to November 2010, 1295 consecutive liver resections for colorectal liver metastases were performed at our Institution. The cohort of caudate resection patients were age ( \pm 1 year), sex and number of resected segments matched with an exact number of other hepatic resection patients that did not include the caudate lobe. Seventy-five patients had CLR. Six patients who had isolated CLR were excluded leaving 69 cases from analysis.

Results ASA score, comorbidity, number of bilateral resections, steatosis and fibrosis did not differ among the cohorts $(p=N S)$. Similarly the number of tumours, size of the largest tumour and resection margins (R0 vs R1) did not differ among the two groups $(\mathrm{p}=\mathrm{NS})$. Post-operative stay (13.5 vs 10.9 days), ICU admissions (16 vs 7), morbidity (sepsis, bile leak, cardio-vascular, respiratory; 26 vs 27), number of patients transfused ( 7 vs 5), hepatic failure (7 vs 6) and 60-days mortality ( 1 vs 2 ) were not significant (CLR vs NCLR, $\mathrm{p}=\mathrm{NS}$ ). Overall survival at 1,3 and 5 years were $85 \%, 57 \%$ and $48 \%$ for CLR and $95 \%, 61 \%$ and $50 \%$ for NCLR respectively. Disease-free survival at 1,3 , and 5 years were $75 \%, 47 \%$ and $47 \%$ for CLR and $85 \%, 63 \%$ and $53 \%$ respectively.

Conclusion Although caudate lobe involvement adds to the technical complexity of hepatic resection, it does not appear to carry increased morbidity and mortality when associated to major liver surgery. The rate of $\mathrm{R} 0$ resection and overall and disease-free survival does not differ between CLR and NCLR.

Competing interests None declared

\section{PTU-084 INCIDENTAL GALLBLADDER CANCER AFTER LAPAROSCOPIC CHOLECYSTECTOMY: TREATMENT AND SURVEILLANCE POLICIES IN THE UK: RESULT OF A SURVEY}

doi:10.1136/gutjnl-2012-302514c.84

G Marangoni, * A Hakeem, G J Toogood, J P A Lodge, R K Prasad. HPB and Transplant Unit, St James' Hospital, Leeds, UK

Introduction Increasingly diagnosis of early gallbladder cancer (GBC) is incidentally made after laparoscopic cholecystectomy (LC). T stage correlates with nodal metastases and potential for residual disease. Although there is no controversy in the treatment of T1a and T2 tumours, there is still no agreement on what constitutes optimal management for $\mathrm{pT} 1 \mathrm{~b}$ and the extent of lymphadenectomy for pT2 tumours. Staging, timing of surgery after LC and treatment of the extrahepatic biliary tree are not standardised.

Methods The aim of this study was to assess the treatment, staging and surveillance protocols of incidentally found GBC after LC among hepatobiliary and upper GI surgeons in the UK in the light of the current published literature. A questionnaire on incidental GBC was devised and sent to the Consultant Surgeons members of the
Association of Upper GI Surgeons of Great Britain and Ireland after approval from the Association of Upper GI Surgeons Committee. There were 13 questions regarding treatment protocols according to T category ( $\mathrm{pT} 1 \mathrm{a} / \mathrm{pT} 1 \mathrm{~b}, \mathrm{pT} 2)$, treatment of previous port sites, staging protocols, surveillance policies, timing of surgery after LC, extent of lymphadenectomy and management of the extrahepatic biliary tree.

Results There were 48 completed questionnaire. Over $80 \%$ would consider simple LC an adequate treatment for T1a tumours. 30\% do not advocate radical surgery for $\mathrm{T} 1 \mathrm{~b}$ and $50 \%$ would not perform regional lymphadenectomy. $5 \%$ would not further treat T2 tumours and $23 \%$ would not add regional lymphadenectomy. Over $50 \%$ would not resect port sites. About $50 \%$ would consider chest and abdomen CT only for staging. About $30 \%$ and $80 \%$ would recommend surveillance for CT or MRI for 2-5 years for T1 and T2 tumours after extended resection respectively. $72 \%$ would advocate surgery as soon as possible. $22.5 \%$ and $20 \%$ would perform coeliac trunk lymphadenectomy if staging confirms positive nodes and routinely excise the extrahepatic biliary tree respectively.

Conclusion The only chance of cure for GBC is complete tumour excision. There is heterogeneity among UK surgeons regarding treatment, staging and surveillance policies. According to the survey, UK practice does not fully comply with published evidence for T1a and T2 categories. Although there is increased evidence that T1b tumours would benefit from extended resection only $50 \%$ would perform a regional lymphnode dissection. Clear guidelines would help standardisation of staging and surveillance policies.

Competing interests None declared.

\section{PTU-085 IMPACT OF TRANSIENT ELASTOGRAPHY (FIBROSCAN) IN THE MANAGEMENT OF PATIENTS WITH LIVER DYSFUNCTION IN A REGIONAL CENTRE IN NORTHERN IRELAND}

doi:10.1136/gutjnl-2012-302514c.85

G P Manikpure, ${ }^{*}$ J Cash, N McDougall. Hepatology, Royal Victoria Hospital, Belfast, UK

Introduction Transient elastography (Fibroscan) is a simple, noninvasive method of assessing liver fibrosis. It is measured by an ultrasound transducer probe and results are expressed in kilo-Pascals $(\mathrm{kPa})$ with liver stiffness values ranging from 2.5 to $75 \mathrm{kPa}$. The results are immediately available and are operator-independent. Liver biopsy has long been the gold standard to evaluate fibrosis. Unfortunately, it is invasive and associated with complications. In addition the accuracy of the histology sample is subject to significant heterogeneity. Our aim was to determine how effective Fibroscan is in reducing the need for liver biopsy.

Methods All patients undergoing Fibroscan during a 1-year period from 1 August 2010 to 31 July 2011 in the Royal Victoria Hospital, Belfast were included. Patients who had biopsy pre-planned for the same day as Fibroscan were excluded. The following data were recorded: demographic data, Fibroscan readings, indication for Fibroscan and whether or not Fibroscan prevented the need for subsequent liver biopsy. All patients had at least 10 valid readings.

Results 66 patients ( 36 male) underwent Fibroscan to evaluate if there was any evidence of advanced fibrosis or cirrhosis. The underlying liver diagnoses were Hepatitis $C(30)$, fatty liver disease (7), primary biliary cirrhosis (7), hepatitis B (6), hereditary haemochromatosis (4), deranged liver function tests (4), alcoholic liver disease (3), orthotopic liver transplant (2) and 1 each for cryptogenic cirrhosis, primary sclerosing cholangitis and hyperferritinaemia. Thirty-nine patients had a normal Fibroscan of whom 37 (95\%) did not require a follow-up liver biopsy. 27 patients had high readings suggesting advanced liver fibrosis or cirrhosis of whom $8(30 \%)$ 